\title{
BMJ Global Health Estimating health opportunity costs in low-income and middle-income countries: a novel approach and evidence from cross-country data
}

\author{
Jessica Ochalek, ${ }^{1}$ James Lomas, ${ }^{1}$ Karl Claxton ${ }^{1,2}$
}

To cite: Ochalek J, Lomas J, Claxton K. Estimating health opportunity costs in lowincome and middle-income countries: a novel approach and evidence from crosscountry data. BMJ Glob Health 2018;3:e000964. doi:10.1136/ bmjgh-2018-000964

Handling editor Seye Abimbola

- Additional material is published online only. To view please visit the journal online (http://dx.doi.org/10.1136/ bmjgh-2018-000964).

Received 19 May 2018 Revised 12 September 2018 Accepted 14 September 2018

Check for updates

C Author(s) (or their employer(s)) 2018. Re-use permitted under CC BY. Published by BMJ.

${ }^{1}$ Centre for Health Economics, University of York, York, UK ${ }^{2}$ Department of Economics and Related Studies, University of York, York, UK

Correspondence to Jessica Ochalek; jessica.ochalek@york.ac.uk

\section{ABSTRACT}

The economic evaluation of healthcare interventions requires an assessment of whether the improvement in health outcomes they offer exceeds the improvement in health that would have been possible if the additional resources required had, instead, been made available for other healthcare activities. Therefore, some assessment of these health opportunity costs is required if the best use is to be made of the resources available for healthcare.

This paper provides a framework for generating countryspecific estimates of cost per disability-adjusted life year (DALY) averted 'thresholds' that reflect health opportunity costs. We apply estimated elasticities on mortality, survival, morbidity and a generic measure of health, DALYs, that take account of measures of a country's infrastructure and changes in donor funding to country-specific data on health expenditure, epidemiology and demographics to determine the likely DALYs averted from a $1 \%$ change in expenditure on health. The resulting range of cost per DALY averted 'threshold' estimates for each country that represent likely health opportunity costs tend to fall below the range previously suggested by WHO of $1-3 \times$ gross domestic product (GDP) per capita. The 1-3× GDP range and many other previous and existing recommendations about which interventions are cost-effective are not based on an empirical assessment of the likely health opportunity costs, and as a consequence, the health effects of changes in health expenditure have tended to be underestimated, and there is a risk that interventions regarded as costeffective reduce rather than improve health outcomes overall.

\section{INTRODUCTION}

Evidence of the expected costs and health effects of making a healthcare intervention available to specific populations in a particular setting and healthcare system (HCS) are typically summarised as incremental cost-effectiveness ratios, which are often expressed as the cost per quality-adjusted life year (QALY) gained or the cost per disability-adjusted life year (DALY) averted. ${ }^{1}$ These measures provide a useful summary of how much additional resource is required to achieve a

\section{Key questions}

What is already known?

- An effective intervention will only improve health outcomes overall if the additional health benefits it offers exceed the health opportunity costs associated with the additional healthcare costs that it imposes.

- The criteria commonly used to judge cost-effectiveness (ie, cost per disability-adjusted life year (DALY) averted 'thresholds') do not reflect evidence of health opportunity costs with the result that decisions and recommendations based on them may reduce rather than improve overall population health.

\section{What are the new findings?}

- Available estimates of the health effect of changes in health expenditure using country-level data can be used to inform country-specific assessments of health opportunity costs by applying estimated mortality effects (elasticities) to country-specific data on baseline epidemiology, demographics and health expenditure.

- A range of plausible estimates of the cost per DALY averted from changes in health expenditure are reported for 97 low-income and middle-income countries.

What do the new findings imply?

- The reported estimates are an evidence-based improvement on the type of norms that have become widely cited and used as 'thresholds' to judge cost-effectiveness, and this framework of analysis can be applied to the results of any study thought to identify plausible effects on mortality of changes in health expenditure, whether they are based on country-level or within country data.

measured improvement in health (the additional cost required to gain one QALY or to avert one DALY). Whether the cost per QALY gained or DALY averted offered by an intervention is judged to be cost-effective requires some explicit or implicit criteria, often referred to as a cost-effectiveness (cost 
per DALY averted) 'threshold' below which the intervention is regarded as worthwhile. However, an effective intervention will only improve health outcomes overall (ie, produce a positive net health benefit) if the additional health benefits it offers exceed the health opportunity costs associated with the healthcare costs that it imposes (whether these must be found from existing commitments or additional expenditure that could have been devoted to other healthcare activities). Therefore, an assessment of health opportunity cost indicates the maximum an HCS can afford to pay for the health benefits that an intervention offers without reducing health outcomes overall. It also reflects the value (the health benefits) of increasing healthcare expenditure.

To ensure that decisions improve rather than reduce health outcomes overall, judgements about cost-effectiveness ought to be founded on evidence of the likely health opportunity costs in the HCS where the use of an intervention is being considered. ${ }^{2}$ A persistent problem has been that the criteria used to judge cost-effectiveness (ie, cost per DALY 'thresholds') recommended or cited by decision making and advisory bodies (both national and supranational) reflect a lack of conceptual clarity about what they ought to represent and what type of evidence might inform their assessment. ${ }^{34}$ These implicit values and established norms do not reflect evidence of health opportunity costs, so decisions or recommendations based on them may reduce rather than improve overall population health. ${ }^{2}$ For example, the cost per DALY averted 'thresholds' previously promoted by the WHO, which classified interventions as very cost effective $(1 \times$ gross domestic product (GDP) per capita or less) and cost-effective (3× GDP per capita or less), became a widely cited and established norm but did not reflect evidence of health opportunity costs. ${ }^{5}$ The explicit and/ or widely cited norms that have become established in some high-income settings are also recognised as having little evidential foundation. ${ }^{6-9}$ Other proposed 'thresholds' reflect a view of what value ought to be placed on improvements in health. They imply what healthcare expenditure ought to be (the social demand for health) rather than an evidence-based assessment of health opportunity costs given actual levels of expenditure, that is, a 'supply side' estimate of the amount of health that an HCS currently delivers with more or less resources. ${ }^{1011}$

The health opportunity cost of a proposed investment in a healthcare intervention is the improvement in health that would have been possible if the additional resources required had, instead, been made available for other healthcare activities. This assessment is equally relevant whether the additional costs of the investment must be found from existing commitments and current levels of health expenditure, or when health expenditure will be increased to accommodate the additional resources required. Therefore, an estimate of the marginal productivity of healthcare expenditure also represents expected health opportunity costs when the decision context is restricted to approving or rejecting a proposed investment. Decision makers may also compare a proposed investment to specific disinvestments required to accommodate it or alternative investments that could be made with any additional resources. However, they still need to consider how these alternatives compare with what the HCS could be expected to deliver, that is, an estimate of marginal productivity is still relevant. If the decision maker had full information about all interventions that are or could be provided for all indications and subgroups of the population and was also tasked with the wholesale redesign of the health care system, then the marginal productivity would be the outcome of this task. There are no examples of this type of wholesale redesign for each proposed investment. There are some limited examples of periodic wholesale package design in low-income settings, but even here some estimate of existing marginal productivity has been shown to be a useful starting point in package design. ${ }^{12}$

Estimates of the marginal productivity of health expenditure in producing health (QALYs) are becoming available for some high-income countries based on approaches to estimation that exploit within-country data. ${ }^{13-16}$ This evidence from national HCS in high-income countries gives some indication of possible values in other contexts based on estimates of the income elasticity of demand for health and assumptions about the relative underfunding of HCS (ie, the shadow price for public expenditure on health) ${ }^{17}$ However, there are estimates of the mortality effects of changes in healthcare expenditure based on country-level data (typically expressed as elasticities) which, in combination with country-specific data on baseline epidemiology, demographics and health expenditure, offer the opportunity to estimate country-specific cost per DALY averted 'thresholds' that reflect evidence of health opportunity costs.

\section{METHODS}

The effect of different levels of healthcare expenditure on mortality outcomes has been investigated in a number of published studies using cross-country data. ${ }^{18}$ The challenge is to control for all the other reasons why mortality might differ between countries in order to isolate the causal effect of differences in health expenditure. ${ }^{19}$ Even if available measures are complete, accurate and unbiased, then estimation issues are likely to occur because of simultaneous equation bias, where health outcomes are likely to be influenced by expenditure (increases in expenditure improves outcomes), but outcomes are also likely to influence expenditure (poor outcomes prompt greater efforts and increased expenditure) ${ }^{20}$ This results in endogeneity which, combined with inevitable aggregation bias, risks underestimating the magnitude of health improvements due to changes in expenditure. Instrumental variables have been used in a number of studies to try and overcome this problem and estimate outcome elasticities for mortality. ${ }^{18}$ This approach requires that the excluded instruments satisfy two criteria. The first is that 
the instruments are relevant, that is, the excluded instruments strongly predict the endogenous instrumented variables. This is typically judged by calculating the f-statistic of a joint test of the excluded instruments where the statistic should be at least $10 .^{21}$ The second criterion is that the instruments are valid, which means that the instruments themselves do not affect the outcome variable directly or through some unobserved factor, but instead only influence the outcome indirectly through their effect on the endogenous instrumented variable. Instrument validity cannot be directly tested, and expert judgement is required, but when an equation is overidentified (there are more excluded instruments than endogenous variables) an overidentification test can be helpful, although it may lack power in rejecting the null hypothesis of joint validity in some cases. ${ }^{22}$

The Bokhari et al model specification applies an instrumental variable approach to cross-sectional data from the year 2000 for 127 countries and models both public expenditure on health and a country's GDP as endogenous variables (both in per capita terms). Their identification strategy employs as instrumental variables: consumption-investment ratio, military expenditure per capita of neighbouring countries and measures of institutional quality. ${ }^{23}$ Bokhari et al make the case that the consumption-investment ratio is related to the level of GDP, but not directly to health outcomes, making it a suitable instrumental variable for GDP. Similarly, they argue that military expenditure in neighbouring countries is a reasonable instrument for public expenditure on health because it is not directly related to health outcomes, and it encourages domestic military expenditure that crowds out other public expenditure, including health. Finally, they argue that GDP and public expenditure on health are related to the institutional quality instruments that reflect economic management and policies for social inclusion and equity, respectively. These are typical instrumental variables following in the tradition of earlier papers and pass the standard tests for relevance and validity. ${ }^{24}$ In addition, Bokhari et al allow for the outcome elasticity with respect to expenditure of countries to vary by two variables reflecting the level of infrastructure and shock in donor funding.
The results from this approach to estimation using cross-country data can inform country-specific cost per DALY averted values by applying estimated elasticities to country-specific mortality rates, conditional life expectancies (CLEs) and population distribution (all by age and gender) as well as estimates of disability burden of disease and total healthcare expenditure. We use the Bokhari et al model specification and expand on their original dataset for year 2000 (ie, under-5 mortality and the original instrumental variables, including bespoke data on institutional quality) to re-estimate the effect of changes in expenditure on adult male and adult female mortality from the World Bank, enabling greater coverage of the population, as well as: (1) a measure of the survival burden of disease: years of life lost (YLLs); (2) a measure of the morbidity burden of disease: years of life disabled (YLDs); and (3) DALYs, a generic measure of overall ill health, from the Global Burden of Disease (GBD) database. ${ }^{25}$ Elasticities are calculated at the country level and differ only with respect to the interaction of measures of infrastructure and donor funding. While data from 2000 is used, there is little evidence to suggest elasticities would be expected to vary over time. ${ }^{18}$ The estimated elasticities for low-income and middle-income coutries (LMICs) (see table 1) are applied to country-specific data from 2015 on health expenditure, epidemiology and demographics.

There are four ways in which the estimated elasticities in table 1 can be used to estimate the likely DALYs averted as a consequence of a $1 \%$ change in health expenditure in each country, $i$. These are summarised in table 2 and are briefly described below, with details of the data used reported in online supplementary file 2.

\section{DALY 1}

The first estimate is based only on estimates of the mortality effects of changes in expenditure. As these are the most prevalent estimates available across the literature, this means that in principle DALY 1 could be calculated using elasticities from various sources, for example, all-cause mortality elasticities estimated from within-country data. ${ }^{26} 27$

The estimated elasticity for children under-5 for each country $i, \epsilon_{i}^{\text {mortality }}{ }^{0-4}$, can be applied to the number of

Table 1 Estimated elasticities for LMICs

\begin{tabular}{llll}
\hline & Average & Minimum magnitude & Maximum magnitude \\
\hline Mortality (deaths per 1000) & & & -0.35 \\
Children under-5 & -0.33 & -0.25 & -0.19 \\
Adults females & -0.17 & -0.08 & -0.20 \\
Adult males & -0.18 & -0.10 & -0.30 \\
YLLs & -0.30 & -0.26 & -0.04 \\
YLDs & -0.03 & -0.02 & -0.21 \\
\hline DALYs & -0.21 & -0.18 & \\
\hline
\end{tabular}

DALYs, disability-adjusted life years; LMICS, low-income and middle-income coutries; YLDs, years of life disabled; YLLs, years of life lost. 
Table 2 Alternative approaches to calculating DALYs averted

\begin{tabular}{|c|c|c|c|c|c|}
\hline & & DALY 1 & DALY 2 & DALY 3 & DALY 4 \\
\hline \multicolumn{2}{|l|}{$\begin{array}{l}\text { Survival effects } \\
\text { (YLLs averted) }\end{array}$} & $\begin{array}{l}\text { Based on indirectly } \\
\text { estimating effects } \\
\text { on survival from } \\
\text { mortality (A). }\end{array}$ & \multicolumn{2}{|c|}{ Directly estimated (D). } & \multirow[t]{3}{*}{$\begin{array}{l}\text { Directly estimated } \\
\text { (G). }\end{array}$} \\
\hline \multirow[t]{2}{*}{$\begin{array}{l}\text { Morbidity effects } \\
\text { (YLDs averted) }\end{array}$} & Direct effect & $\begin{array}{l}\text { Uses indirectly } \\
\text { estimated effects } \\
\text { on survival from } \\
\text { mortality as a } \\
\text { surrogate for } \\
\text { morbidity effects } \\
\text { (B). }\end{array}$ & $\begin{array}{l}\text { Uses directly } \\
\text { estimated } \\
\text { survival effects } \\
\text { as a surrogate for } \\
\text { morbidity effects } \\
\text { (E). }\end{array}$ & \multirow[t]{2}{*}{$\begin{array}{l}\text { Directly estimated } \\
\text { (F). }\end{array}$} & \\
\hline & Indirect effect & \multicolumn{2}{|c|}{$\begin{array}{l}\text { Uses average overall population health } \\
\text { as a surrogate for increase in YLD } \\
\text { burden associated with increase in YLLs } \\
\text { averted (C). }\end{array}$} & & \\
\hline
\end{tabular}

DALYs, disability-adjusted life years; YLDs, years of life disabled; YLLs, years of life lost.

deaths observed in this age group in each country to provide an estimate of the number of under-5 deaths averted as a consequence of a $1 \%$ change in country health expenditure.

$$
\text { directly estimated deaths averted }{ }_{i}^{0-4}=1 \% *\left|\epsilon_{i}^{\text {mortality }^{0-4}}\right| * \text { deaths }_{i}^{0-4}
$$

Similarly, the estimated elasticities for male and female adults (ages 15-60 years) are applied to observed deaths by age and gender in each country, that is, assuming that the proportionate effect on mortality applies equally across age groups within 15-60 years age range.

$$
\begin{aligned}
& \text { directly estimated deaths averted }{ }_{i}^{15-60}=1 \% *\left|\epsilon_{i}^{\text {mortality }}{ }^{15-60}\right| * \text { deaths }_{i}^{15-19} \\
& +\ldots+1 \% *\left|\epsilon_{i}^{\text {mortality }} 15-60\right| * \text { deaths }_{i}^{55-60}
\end{aligned}
$$

Once the likely deaths averted by a $1 \%$ change in health expenditure have been estimated (see Eqs. (1) and (2)), the survival effects can be established by applying CLE at age of death to each death averted within each age group for which deaths averted have been estimated (see Eq. (3)) and scaling these survival effects to be for the whole population using data on the population level YLL burden (see Eq. (4)). This assumes that the survival effects of changes expenditure are in proportion to the survival burden of disease in each age group.

$$
\begin{aligned}
\text { mortality based YLL averted }_{i}^{0-4} \& 15-60 & =C L E_{i}^{0-4} * \text { deaths averted }_{i}^{0-4} \\
& +C L E_{i}^{15-19} * \text { deaths averted } d_{i}^{15-19} \\
& +C L E_{i}^{20-24} * \text { deaths averted } d_{i}^{20-24} \\
& +\ldots \\
& +C L E_{i}^{55-59} * \text { deaths averted }_{i}^{55-59}
\end{aligned}
$$

$$
\begin{aligned}
& \text { mortality based } Y L L \text { averted }_{i}^{\text {all ages }} \\
& =\text { mortality based } Y L L \text { averted }_{i}^{0-4} \& 15-60 / \frac{Y L L_{i}^{0-4}+Y L L_{i}^{15-60}}{Y L L_{i}^{\text {all ages }}}
\end{aligned}
$$

Changes in expenditure that affect mortality and survival are also likely to have an effect on morbidity through the prevention and treatment of disease (ie, a direct effect decreasing YLD burden). However, an indirect effect may also be present as reductions in mortality and the resulting increased survival is likely to increase the number of years during which morbidity is experienced.

To calculate the possible direct effect, we assume that the effect of changes in expenditure on morbidity is proportional to the effect on survival ( $B$ in table 2 ), that is, assuming that the estimated effects on mortality can be used as a surrogate for likely effects on morbidity where these effects have not been directly estimated. The ratio of YLD to YLL in each country is applied to estimates of the country-specific survival effects from Eq. (4). To account for the indirect effect of increasing the number of years during which morbidity is experienced due to the survival effects, we apply the per capita YLD burden for each country to the country-specific survival effects (see the second term in Eq. (5) below and C in table 2). Mortality based YLD averted are therefore calculated as:

$$
\begin{aligned}
& \text { mortality based } Y L D \text { averted }_{i}^{\text {all ages }}=\text { mortality based YLL averted }_{i}^{\text {all ages }} \\
& * \frac{Y L D_{i}^{\text {all ages }}}{Y L L_{i}^{\text {all ages }}} \\
& \text { - mortality based YLL averted } d_{i}^{\text {all ages }} \\
& \text { * per capita YLD } \text { burden }_{i}
\end{aligned}
$$

The total DALYs averted due to a $1 \%$ change in health expenditure in each country is the sum of the survival effects (the YLL averted in Eq. (4), A in table 2) and the net morbidity effects (YLD averted in Eq. (5), B minus $\mathrm{C}$ in table 2). This illustrates how estimates of mortality effects of health expenditure, in the form of elasticities, can be used to provide an indication of the likely survival and morbidity effects. The health effects of a $1 \%$ change in country health expenditure will differ by country due to differences in the number observed deaths by age and 
gender and differences in age-specific and gender-specific CLE as well as elasticities. The amount of expenditure required to avert one DALY will also differ by country due to differences in total health expenditure.

$$
\text { cost per DALY averted }{ }_{i}=\frac{1 \% * \text { government expenditure on health }_{i}}{D A L Y \text { averted }_{i}}
$$

Nonetheless, a key assumption has been required, which is that the estimated mortality and survival effects of changes in health expenditure are a good surrogate for the morbidity effects.

\section{DALY 2}

The effect of changes in health expenditure on measures of survival burden of disease can also be estimated directly from the cross-country data (see table 1 ). The estimated elasticity for YLL, $\epsilon_{i}^{Y L L}$, can be applied to country-specific $Y L L_{i}^{\text {all ages }}$, which are calculated from observed mortality and CLE by age and gender. Therefore, YLLs averted due to a $1 \%$ change in health expenditure can be directly estimated (Eq. 7) rather than applying CLE to estimates of deaths averted by age and gender (as required in Eqs. (1) to (4) above).

$$
\text { directly estimated } Y L L \text { averted }_{i}=1 \% *\left|\epsilon_{i}^{Y L L}\right| * Y L L_{i}^{\text {all ages }}
$$

The possible direct and indirect effects on morbidity of changes in health expenditure that affects survival can be calculated in the same way as previously; assuming that that the estimated effects on survival can be used as a surrogate for likely effects on morbidity and with the indirect effect of increases in morbidity based on directly estimated survival effects. Therefore, the net morbidity effects are calculated in the same way as in Eq. (5) but with directly estimated $Y L L_{i}$ averted replacing mortality based $Y L L_{i}$ averted (E minus $\mathrm{C}$ in table 2).

\section{DALY 3}

As well as direct estimates of the effect on survival burden of disease, the effect of changes in health expenditure on measures of morbidity burden of disease (YLD) can also be estimated directly from the cross-country data (see table 1). The estimated elasticity for YLD, $\epsilon_{i}^{Y L D}$, is applied to country-specific $Y L D_{i}^{\text {all ages }}$, which is available at the country level from GBD. ${ }^{25}$

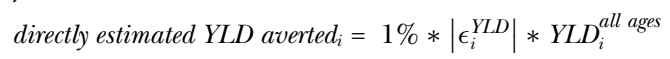

DALY 3 uses direct estimates of the effect on survival burden in the same way as DALY 2 but combines these with direct estimates of the effect on morbidity. The total DALYs averted due to a $1 \%$ change in health expenditure in each country is the sum of the directly estimated survival effects (YLL averted in Eq. (7), D in table 2) and the directly estimated morbidity effects (YLD averted in Eq. (8), $\mathrm{F}$ in table 2).

\section{DALY 4}

The combined effect of changes in expenditure on survival and morbidity burden of disease (DALYs) can be estimated directly from the cross-country data using country-level estimates of DALY burden of disease.
Country-specific estimates of DALY burden (DALY $Y_{i}^{\text {all ages }}$ ) are calculated as the sum of $Y L L_{i}^{\text {all ages }}$ and $Y L D_{i}^{\text {all ages }}$ for each country $i$. Therefore, a direct estimate of DALYs averted for a $1 \%$ change in provincial health expenditure is simply the product of the estimated DALY burden for that country and the estimated elasticity $\epsilon_{i}^{D A L Y}$ (Eq. 9).

$$
\text { directly estimated DALY averted } \text { a }_{i}=1 \% *\left|\epsilon_{i}^{D A L Y}\right| * D A L Y_{i}^{\text {all ages }}
$$

\section{Summary}

These four alternative ways to estimate health opportunity costs, as measured by the cost per DALY averted, make slightly different assumptions. The comparison of DALY 1 with DALY 4 gives some indication of whether it is reasonable to use estimates of the mortality effect of changes in health expenditure as a surrogate for likely survival and morbidity effects. This finding is itself useful for studies with estimated elasticities for mortality outcomes but requiring additional information about the effect of expenditure on other health outcomes. In particular, studies investigating the relationship between mortality and health expenditure using high-quality within-country data (typically undertaken in high-income countries), which overcomes some of the difficulties and challenges of estimation based on aggregate country-level data.

\section{RESULTS}

\section{Estimated elasticities for LMICs}

The extended Bokhari et almodel generated country-specific elasticity results for all of the countries in the model, where the elasticities differed due to the specification of the relationship of expenditure with health. The average and range of elasticities for each of the six measures of health outcome are reported in table 1.

Estimated elasticities differ due to the presence of interaction terms combining spending and level of infrastructure (proxied by 'paved roads per unit of area') and the absolute deviation in donor funding from the historical mean. The direction and size of the difference in elasticities between countries depends on the signs of the estimated coefficients on the interaction terms and relative magnitude of each of these.

\section{Cost per DALY averted}

The estimates of cost per DALY averted for each country are reported in online supplementary file 1 where they are also expressed as \% of GDP per capita. Few countries have any estimates that are higher than $1 \times$ GDP per capita and none are higher than $3 \times$ GDP per capita. Among those with any estimate higher than 1× GDP per capita, all are middle-income countries with slightly lower average mortality, survival and ill health burdens than LMICs on average.

In almost all cases, DALY 2 provides the lowest cost per DALY averted estimate for each country. This reflects the fact that the estimated elasticity for survival effects (YLL) 


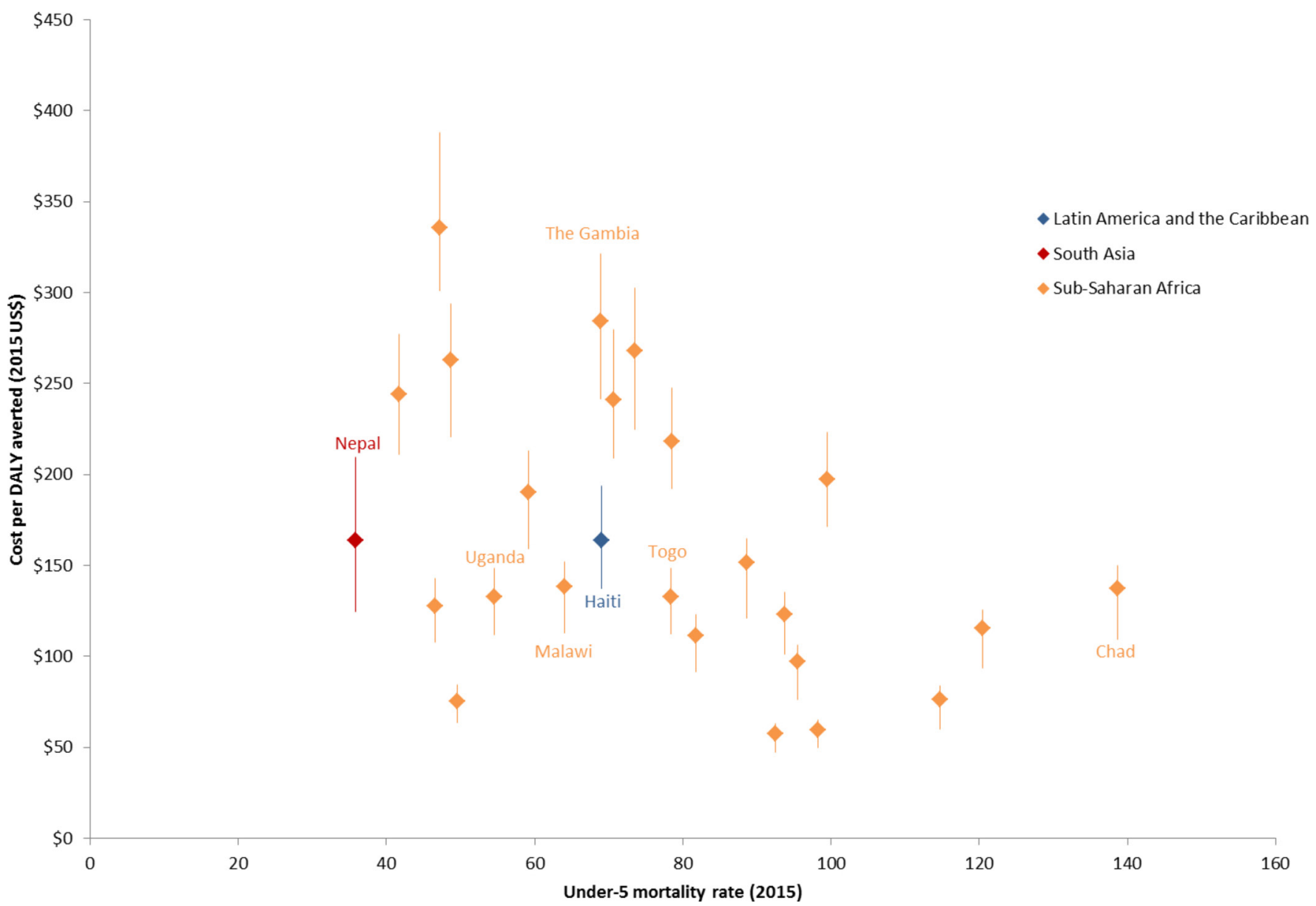

Figure 1 Cost per DALY averted by under-5 mortality rate for low-income countries. DALY, disability-adjusted life year.

is typically greater than for morbidity effects (YLD) and effects on DALYs. This larger effect on survival is then used as a surrogate for morbidity effects when estimating DALY 2. Although the differences in the elasticities reported in table 1 might indicate that employing this 'surrogacy' assumption risks overestimating morbidity effects, this should not be overinterpreted as the estimated elasticities are not based on within-country data but country-level data. However, in general, the comparison of DALY 1 and DALY 4, which are found to be relatively similar compared with comparing DALY 2 and DALY 3, does suggest that using estimates of the mortality effect of changes in health expenditure as a surrogate for both likely survival and morbidity effects may not be unreasonable although with additional uncertainty.

Figures 1 and 2 illustrate the range of estimates for each low-income country and middle-income country by under-5 mortality rate, respectively. The average of the range of values for each country is not the average for the four cost per DALY averted ratios but the ratio of a $1 \%$ change in expenditure to the average DALYs averted across these four estimates. A pattern is evident between mortality rate and cost per DALY averted. While the low under-5 mortality in Haiti would, other things equal tend to increase the cost per DALY averted; it is higher in The Gambia than in Haiti, which has the same under-5 mortality rate, primarily because The Gambia has higher government expenditure on health. This is also illustrated by Panama and El Salvador as well as Congo and Namibia.

Figure 3 illustrates the same cost per DALY averted estimates but now by per capita government expenditure on health. It suggests that the cost per DALY averted increases with per capita health expenditure which is, in general, what might be expected, although this is to some extent inevitable given the methods used to generate these estimates. It also illustrates the similarity in the range of estimates for most countries but also why others differ. For example, although Russia has nearly double the per capita government expenditure on health of Malaysia and has a similar under-5 mortality rate (see figure 2), it has higher baseline adult mortality as well as YLD, YLL and DALY burden than Malaysia and therefore a lower cost per DALY averted range than might otherwise be expected.

However, although Cape Verde and Congo have very similar per capita expenditure on health, our range of estimates of cost per DALY averted for Cape Verde is \$1938-\$2843 compared with \$1235-\$1613 in Congo. Part of the difference is due to the baseline mortality rates in Congo, which are more than double those in Cape Verde. Both countries also differ in terms of the 


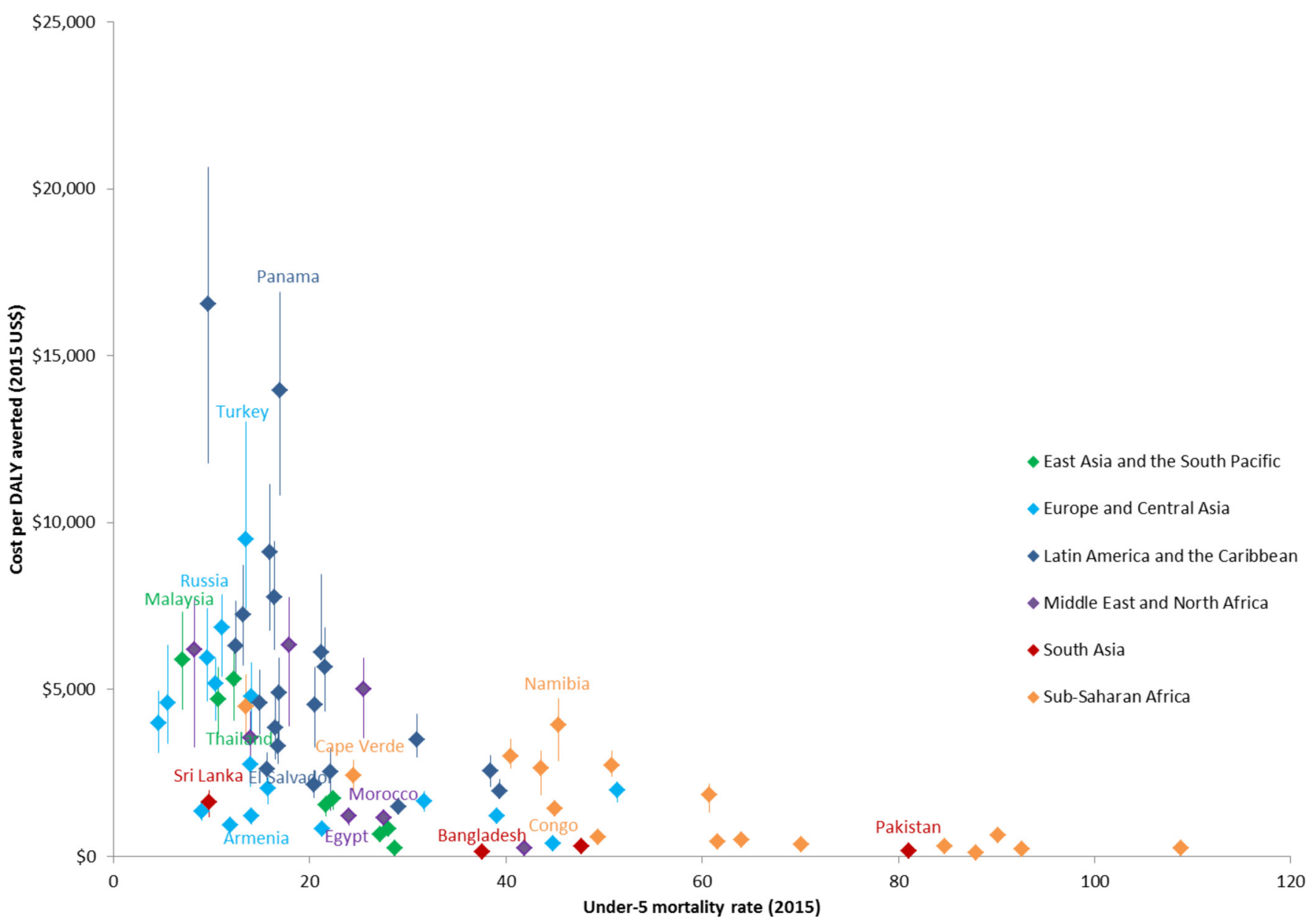

Figure 2 Cost per DALY averted by under-5 mortality rate for middle-income countries. DALY, disability-adjusted life year.

age and gender structures of the population, with Congo having a higher per cent of the population in the under-5 age group. These factors all contribute to the differences in cost per DALY averted between the two countries and are relevant to cost per DALY averted for all countries.

The apparent similarity in the range of cost per DALY averted between countries should not be overinterpreted as estimates would also be expected to differ if countries are able to generate health at different rates, which would be reflected in differing elasticities. The ranges of estimated elasticities for each country vary little, reflecting the limited economic significance of the estimated coefficients on the interaction terms. This underscores the importance of further research using within-country data to estimate these values at a country level and to account for the heterogeneity between different HCS.

\section{DISCUSSION}

Estimates of the health opportunity costs of healthcare expenditure are critical for informing assessments of whether the improvement in health outcomes offered by investing additional resources in a new intervention exceeds the improvement in health that would have been possible if the additional resources required had, instead, been made available for other healthcare activities. Commonly established implied norms, such as 1-3× GDP per capita, are often inappropriately applied in practice to judge cost-effectiveness. ${ }^{5}{ }^{28}$ Such values generally reflect norms or the social demand for health (ie, a view of what value ought to be placed on improvements in health) rather than an evidence-based assessment of health opportunity costs given actual levels of expenditure. ${ }^{29}$ As such, they do not reflect the health that the HCS is currently able to deliver with the resources available, that is, the 'supply side' of the HCS. Adopting 'thresholds' to judge cost-effectiveness that are too high and do not reflect the 'supply side' will lead to decisions that reduce overall health because the health gained from adopting an intervention will be more than offset by the health opportunity costs elsewhere in the HCS. As well as leading to net harms for population health, it may also exacerbate health inequalities and unwarranted variations in access to other healthcare, depending on where the health opportunity costs of additional healthcare costs tend to fall.

The framework of analysis set out in this report illustrates how estimates of the relationship between mortality and variations in healthcare expenditure can be employed alongside country-specific data on demography, epidemiological profile and expenditure to inform estimates 


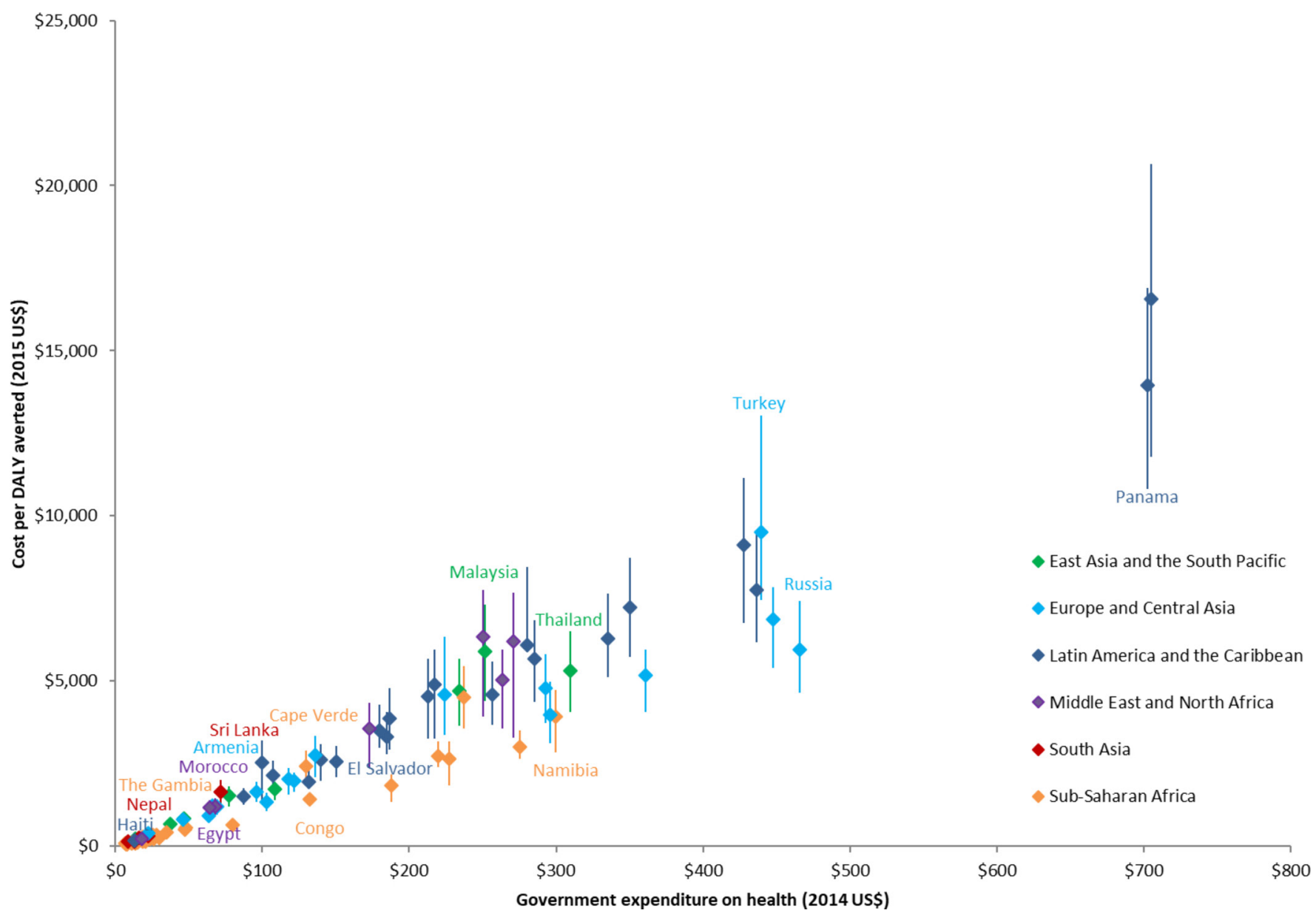

Figure 3 Cost per DALY averted by per capita government expenditure on health for LMICs. DALY, disability-adjusted life year.

of health opportunity costs. While data are readily available for the latter, reliable estimates of the relationship between mortality and variations in healthcare expenditure present a challenge.

This paper employed estimates from the model used by Bokhari et al, which applies an instrumental variable method to cross-sectional data, and models both public expenditure on health and a country's GDP as endogenous variables. While Bokhari et al find a statistically and economically significant effect of public expenditure on health-reducing mortality outcomes, there is no clear and consistent finding in the literature that evaluates the relationship between mortality and variations in healthcare expenditure using cross-country data. ${ }^{18}$ This is often driven by the methodological approach adopted by each study, addressing the considerable challenges including the important country-level heterogeneity, much of which is unobserved and uncontrolled for using existing data, even if it is assumed that systematically unbiased measurements are available. Estimates of mortality elasticities based on cross-country data may be lower than those based on within-country data reflecting the greater dangers of aggregation bias using country-level data and the difficulty of fully accounting for unobserved heterogeneity and endogeneity using the instruments for health expenditure that are available across countries. Irrespective of the level of aggregation analysed, econometric modelling-like all modelling-requires assumptions to be made. Of particular relevance is the assumption of instrument validity when using an instrumental variable approach, which cannot be tested directly. In addition, econometric models may be to some extent fragile to small changes in how data are defined or whether the data are log-transformed prior to analysis. ${ }^{19}$

The framework of analysis employed here can be applied to the results of any econometric study that is thought to identify plausible effects on health outcomes of changes or differences in health expenditure. It can equally be applied to the point estimates of elasticities as it can to percentiles of the uncertain elasticity estimates or to scenarios concerning possible elasticity values. Within-country studies, limited currently, however, to high-income countries, have estimated the marginal productivity of health expenditure in producing health (QALYs). ${ }^{13-16}$ This kind of study requires high-quality data on health outcomes and expenditures by subnational areas, potentially over time, in addition to information to control for healthcare need and to form the basis of instrumental variables. The implied all-cause mortality elasticity estimate, -1.0278 , found by Claxton 
et al is considerably higher in magnitude to any of the mortality elasticity estimates from the extended Bokhari et al model. Another study, Andrews et al used an alternative approach to identification to directly estimate an all-cause mortality elasticity estimate for the UK National Health Service of -0.705 . Once again, this is higher than the results from Bokhari et al. A comparable estimate for Australia reveals a much larger all-cause mortality elasticity of $-2.190 .^{30}$ Other similar studies have been undertaken in the contexts of Australia and Spain, and while the overall results in terms of expenditure per QALY give similar results to these UK studies, the elasticities cannot be directly compared due to their estimations employing a different dependent variable. ${ }^{1416}$

The ranges estimated here are consistent with the ranges of implied cost per QALY gained for countries based on the analysis in Woods et al, which extrapolates the UK findings based on estimates of the income elasticity of demand for health and assumptions about the relative underfunding of HCS (ie, the shadow price for public expenditure on health). An assessment that elasticities using within-country data are likely to be higher than those based on country-level data is plausible and tends to be supported by growing literature from other countries, in particular the studies set in the UK and Australia contexts discussed above. Nonetheless, further research to provide elasticity estimates using within-country and within-state or province data where applicable would be welcome.

\section{CONCLUSION}

Few LMICs are likely to have access to the type of within-country data that could be used to directly estimate their cost per DALY averted. This paper demonstrates that it is possible to generate country-specific estimates by applying elasticities estimated from cross-country data to country-specific baseline data. This offers country-specific approximations based on an underlying international health production function. Nevertheless, the resulting range of country-specific estimates are more likely to indicate the scale of health opportunity costs than previously applied norms that have become widely cited. Therefore, they could be used as interim guidance while research on within-country research is developed. In doing so, it should be noted that where budgets for health are devolved to states or provinces and where there are differences in demography and epidemiology, there are also likely to be differences in health opportunity costs by geography within a country.

The more fundamental contribution of this paper is to clarify the often confused concept of a 'threshold' by demonstrating that judgements about cost-effectiveness can be informed by an empirical assessment of the likely health opportunity costs faced, given existing levels of health expenditure. The demonstration of the type of empirical analysis that can support this assessment also makes this concept a real and practical way to help inform better decision making in LMICs and influence how supranational bodies make recommendations and set priorities, including purchasing and investment decisions. These continuing research efforts start to identify the real value of devoting more resources to healthcare and can contribute to greater accountability for the healthcare and other expenditure decisions made at a local, national and supranational levels.

Contributors All authors contributed to the concept and design of this paper. J0 led on the analysis and drafting, and the final paper was edited and approved by all authors.

Funding This study was funded by http://dx.doi.org/10.13039/100000865 Bill and Melinda Gates Foundation (Grant Number: OPP1165566).

Competing interests None declared.

Patient consent Not required.

Provenance and peer review Not commissioned; externally peer reviewed.

Data sharing statement № additional data are available.

Open access This is an open access article distributed in accordance with the Creative Commons Attribution 4.0 Unported (CC BY 4.0) license, which permits others to copy, redistribute, remix, transform and build upon this work for any purpose, provided the original work is properly cited, a link to the licence is given, and indication of whether changes were made. See: http://creativecommons.org/ licenses/by/4.0/.

\section{REFERENCES}

1. Salomon JA, Vos T, Hogan DR, et al. Common values in assessing health outcomes from disease and injury: disability weights measurement study for the global burden of disease study 2010. Lancet 2012;380:2129-43.

2. Claxton K, Sculpher M, Palmer S, et al. Causes for concern: is NICE failing to uphold its responsibilities to all NHS patients? Health Econ 2015;24:1-7.

3. Revill P, Walker S, Madan J, 2014. Using cost-effectiveness thresholds to determine value for money in low- and middle-income country healthcare systems: are current international norms fit for purpose? York. https://www.york.ac.uk/media/che/documents/ papers/researchpapers/CHERP98_costeffectiveness_thresholds value_low_middle_income_countries.pdf (accessed 27 Nov 2015).

4. Culyer AJ. Cost-effectiveness thresholds in health care: a bookshelf guide to their meaning and use. Health Econ Policy Law 2016;11:415-32

5. Bertram MY, Lauer JA, De Joncheere K, 2016. Use and misuse of thresholds cost-effectiveness thresholds: pros and cons. Bull world health organ. http://www.who.int/bulletin/online_first/en/ (accessed 6 Oct 2016).

6. Appleby J, Devlin N, Parkin D, et al. Searching for cost effectiveness thresholds in the NHS. Health Policy 2009;91:239-45.

7. H.o.C.H. Committee.. First report of the health committee 20072008. London, 2008.

8. Rawlins MD, Culyer AJ. National Institute for clinical excellence and its value judgments. BMJ 2004;329:224-7.

9. Neumann PJ, Cohen JT, Weinstein MC. Updating cost-effectiveness-the curious resilience of the $\$ 50,000-$ per-QALY threshold. N Engl J Med 2014;371:796-7.

10. Vallejo-Torres L, García-Lorenzo B, Castilla I, et al. On the estimation of the cost-effectiveness threshold: why, what, how? Value Health 2016;19:558-66.

11. Thokala P, Ochalek J, Leech AA, et al. Cost-effectiveness thresholds: the Past, the Present and the Future. Pharmacoeconomics 2018;36:509-22.

12. Ochalek J, Revill P, Manthalu G, et al. Supporting the development of a health benefits package in Malawi. BMJ Glob Health 2018;3:e000607.

13. Martin S, Rice N, Smith PC. Does health care spending improve health outcomes? Evidence from english programme budgeting data. J Health Econ 2008;27:826-42.

14. Edney LC, Haji Ali Afzali H, Cheng TC, et al. Estimating the reference incremental cost-effectiveness ratio for the Australian Health System. Pharmacoeconomics 2018;36:239-52. 
15. Claxton K, Martin S, Soares M, et al. Methods for the estimation of the National Institute for Health and Care Excellence costeffectiveness threshold. Health Technol Assess 2015;19:1-504. v-vi. doi.

16. Vallejo-Torres L, García-Lorenzo B, Serrano-Aguilar P, 2016. Estimating a Cost-effectiveness threshold for the Spanish NHS. Madrid. http://documentos.fedea.net/pubs/eee/eee2016-22.pdf. (accessed 31 Jul 2017).

17. Woods B, Revill P, Sculpher M, et al. Country-level costeffectiveness thresholds: initial estimates and the need for further research. Value Health 2016;19:929-35.

18. Gallet CA, Doucouliagos $\mathrm{H}$. The impact of healthcare spending on health outcomes: a meta-regression analysis. Soc Sci Med 2017;179:9-17.

19. Nakamura R, Lomas J, Claxton K, 2016. Assessing the impact of health care expenditures on mortality using cross-country data. York. https://www.york.ac.uk/media/che/documents/papers/ researchpapers/CHERP128_health_care_expenditures_mortality_ cross-country_data.pdf (accessed 3 May 2017).

20. Gravelle HS, Backhouse ME. International cross-section analysis of the determination of mortality. Soc Sci Med 1987;25:427-41.

21. Staiger D, Stock JH. Instrumental Variables Regression with Weak Instruments. Econometrica 1997;65:557-86.

22. Kovandzic T, Schaffer ME, Kleck G. Estimating the causal effect of gun prevalence on homicide rates: a local average treatment effect approach. J Quant Criminol 2013;29:477-541.
23. Bokhari FA, Gai $Y$, Gottret P. Government health expenditures and health outcomes. Health Econ 2007;16:257-73.

24. Filmer D, Pritchett $L$. The impact of public spending on health: does money matter? Soc Sci Med 1999;49:1309-23.

25. Institute for Health Metrics and Evaluation, 2018. Global Burden of Disease Study 2015 (GBD 2015) Data Resources | GHDx. http:// ghdx.healthdata.org/gbd-2015. (accessed 21 Mar 2018).

26. Claxton K, Lomas J, Martin S, 2017. Estimating Expected Health Opportunity Costs in the NHS (Analysis of 2012/13 Expenditure Data). York. https://www.york.ac.uk/media/che/documents/ Estimating_expected_health_opportunity_costs_in_the_NHS 201213.pdf (accessed 23 Jan 2018).

27. Andrews M, Elamin O, Hall AR, et al. Inference in the presence of redundant moment conditions and the impact of government health expenditure on health outcomes in England. Econom Rev 2017;36:23-41.

28. Leech AA, Kim DD, Cohen JT, et al. Use and Misuse of costeffectiveness analysis thresholds in low- and middle-income countries: trends in cost-per-daly studies. Value Health 2018;21:759-61.

29. Jamison DT, Gelband H, Horton S. Disease control priorities: improving health and reducing poverty. Washington D.C: World Bank, 2018.

30. Edney LC, Haji Ali Afzali H, Cheng TC, et al. Mortality reductions from marginal increases in public spending on health. Health Policy 2018;122:892-9. 
Correction: Estimating health opportunity costs in lowincome and middle-income countries: a novel approach and evidence from cross-country data

Ochalek J, Lomas J, Claxton K. Estimating health opportunity costs in low-income and middle-income countries: a novel approach and evidence from cross-country data. BMJ Global Health 2018;3:e000964. doi: 10.1136/bmjgh-2018-000964

The license type for this paper has changed from CC BY-NC to CC BY.

Open access This is an open access article distributed in accordance with the Creative Commons Attribution 4.0 Unported (CC BY 4.0) license, which permits others to copy, redistribute, remix, transform and build upon this work for any purpose, provided the original work is properly cited, a link to the licence is given, and indication of whether changes were made. See:https://creativecommons.org/licenses/by/4.0/

C Author(s) (or their employer(s)) 2019. Re-use permitted under CC BY. Published by BMJ.

BMJ Global Health 2019;4. doi:10.1136/bmjgh-2018-000964corr1

A) Check for updates 